\title{
Improving cardiac assessment and monitoring in methadone prescribing at an addiction service
}

\author{
Mark Ramage, Kate Fadaka \\ Barnet Drug and Alcohol Service. Barnet, Enfield and Haringey Mental Health NHS Trust
}

\begin{abstract}
The assessment and monitoring of cardiac risk in patients prescribed methadone is important for patient safety. Although uncommon, QTc interval prolongation is potentially life-threatening. Known risk factors for QTc prolongation include methadone, particularly at doses above 100 milligrams daily. This project aimed to improve the cardiac assessment and monitoring at Barnet Drug and Alcohol Service through raising the awareness of this clinically importance issue as well as through amending the medical assessment guidance to promote the comprehensive assessment of QTc prolongation risk factors and ensure the identification of cardiac risk in patients prescribed methadone.
\end{abstract}

The project also provided guidance on QTc prolongation management and monitoring (including performance of an electrocardiogram) at the service to provide a baseline measurement and arrangement of annual monitoring. Prior to the intervention, a randomised sample of 26 patients that had been prescribed methadone revealed no comprehensive cardiac risk assessments. Analysing 52 medical assessments in the month following the intervention suggested an improvement in assessment and management of QTc interval prolongation risk, with seven comprehensive cardiac risks assessments performed and one patient having QTc interval prolongation identified following an electrocardiogram and appropriately managed. This project was limited by small sample sizes and the relatively low incidence of QTC prolongation. The poor rate of attendance for requested electrocardiograms in this population was noted as a challenge that needed further consideration.

\section{Problem}

The Barnet Drug and Alcohol Service (BDAS) is a community addiction service in London in the United Kingdom. BDAS provides a cardiac assessment and monitoring clinic, although patients being prescribed methadone rarely attend. It is important for individuals prescribed methadone to receive appropriate cardiac assessment and monitoring due to the associated risk of prolonged cardiac QTC interval which may lead to sudden death.

\section{Background}

Methadone is associated with electrocardiogram (ECG) changes such as QTc interval prolongation, which is a risk factor for the often fatal ventricular arrhythmia torsades de pointes. Methadone alone may prolong the QTc particularly at doses over 100 milligrams daily or when concurrent with other QTc prolongation risk factors. Such other risk factors include heart or liver disease, family history of QTC prolongation, electrolyte abnormalities, and medications known for QTc prolongation such as citalopram, tricyclic antidepressants, and most antipsychotics.[1] Maudsley Prescribing Guidelines recommend careful monitoring of patients prescribed methadone with risk factors for QTc interval prolongation, in particular for doses of methadone over 100 milligrams of daily.[1] These recommendations ideally comprise a baseline assessment with ECG measurement of the QTc interval and subsequent annual monitoring.[1]

\section{Baseline measurement}

The quality of cardiac assessment and monitoring for patients at BDAS prescribed methadone was investigated by considering a randomised sample whose medical records were analysed according to a series of criteria. The first criteria considered the extent to which the initial medical assessment covered QTc prolongation risk factors. The next criteria considered whether a medical review had occurred within the last year. For patients prescribed methadone with an identified QTc prolongation risk, the final criteria analysed the cardiac risk management and monitoring.

A sample of 49 patients was randomly selected from the 482 under the care of BDAS. Of this sample, 26 were prescribed methadone treatment. On analysing the medical records of these 26 patients regarding their initial medical assessment, no patients had documentation of an assessment covering the full range of QTC prolongation risk factors. Eight patients had some QTc risk factors assessed and two patients had no QTc risk factors assessed. The remaining 16 patients had no documentation of this initial medical assessment available on the electronic medical records due to the date that records were first transferred over to be stored electronically. The assessments classified as covering some QTC risk factors had general documentation of no past medical history or medications and did not evidence specific assessment of QTc risk factors.

The second criteria considered the extent of QTc risk factor assessment at the medical review occurring within the last year for this sample of 26 patients. There were no documented assessments of QTc risk factors for 14 patients, while the other 12 patients had some QTc risk factors explored. Of these 12 patients 
BMJ Quality Improvement Reports

with some QTc risk factors explored, seven patients had no positive findings of QTc risk while the other five patients had QTC prolongation risk identified.

A further analysis was undertaken of the medical reviews for the sample of 26 patients prescribed methadone. This criterion considered whether particular QTc risk factors documented within medical reviews were clearly identified as thereby presenting a QTC prolongation risk which required addressing clinically. From the medical records for these 26 patients, seven showed QTc interval risk factors documented, however only two of these patients were then identified as being at risk of QTc prolongation with a need to clinically address this cardiac risk.

The criteria relating to the management of identified QTc prolongation risk showed that these two patients had an appropriate clinical management plan documented where a QTc risk was identified and documented as such. These plans both happened to include stopping a medication, other than methadone, with a risk of QTc prolongation.

The final criteria considered the monitoring of cardiac risk. This showed that only one of the two patients with QTc risk identified had evidence of referral to the BDAS cardiac assessment clinic for an ECG, and had a management plan including further regular monitoring.

\section{Design}

The initial new medical assessment and subsequent medical reviews performed by clinicians are guided by a new medical assessment document. This reference document consists of a series of important sections that are clinically important for the assessor to cover. This document was lacking a specific area that was focused on cardiac risk factors, with no list of the specific risk factors for QTc interval prolongation. Therefore, the initial intervention amended this document to include a section covering cardiac risk with a reference list of the range of specific QTc risk factors. It was noted from feedback from clinicians after the initial implementation that it would be useful to also include a clear question for clinicians to address whether the patient presented a cardiac risk or not. It also needed to provide recommendations for management for those patients who did have this risk identified.

After editing the document several times in response to feedback from clinicians, the final version comprised of a checklist of the main specific QTc risk factors along with a more detailed list alongside these factors for reference. For example, an item of the checklist to be checked off (whether it had been assessed or not) related to the patient being currently prescribed any medication with a QTC prolongation risk. Alongside this 'yes or no' checkbox was a detailed list of the medications to consider, subdivided within their medication classes for ease of reference. The checklist also included a family history of heart disease, including the details specifically assessing for a family history of QTc interval prolongation. It included heart disease, specifically bradycardia, ischaemic heart disease, and myocardial infarction. It also included liver disease and electrolyte abnormalities as can be seen in alcohol dependency.

The document was also amended to include recommendations in case of identified cardiac risk. This guidance included performance of a mandatory baseline ECG, including measurement of the QTC interval and the arrangement of annual monitoring of cardiac risk through future ECGs at the cardiac assessment clinic.

Amended editions of the document were disseminated as the standard reference for medical assessments at BDAS, with opportunities for amendments to be made in response to feedback from clinicians. It was expected that it would take months to observe any impact of the interventions due to the number of medical assessments of new patients requiring methadone, as well as the relatively low incidence of QTc risk factors and QTc interval prolongation.

\section{Strategy}

Several design iterations of the intervention were required leading up to the final edition of the new medical assessment document. The document was reviewed by prescribers at BDAS at each stage. Difficulties encountered in the stages of the project included making sure that the dissemination of new editions coincided with checking that older editions were not in circulation. It proved vital to emphasise the importance of the amendments in the document so that these were utilised in new medical assessment. This meant communicating with experienced prescribers who were used to not needing to refer to the new medical assessment document very often, particularly regarding highlighting the additional cardiac risk sections and promoting their usage.

Awareness of the background for this project and the intervention being introduced was raised through a variety of means, including emails, presentations, and teaching sessions in the multidisciplinary team meetings. This ensured that the project was communicated to all levels of staff at BDAS as well as engaging the full range of prescribers at the BDAS prescribers meeting. Posters were also produced that briefly detailed the importance of QTc risk in methadone prescribing, along with the interventions introduced aiming to improve cardiac assessment and monitoring. This was in addition to the checklist and reference material regarding QTc risk factors, and the guidance for management of patients prescribed methadone with identified QTc prolongation risk.

\section{Post-measurement}

After the final edition of the intervention had been in place for several months, the potential impact of the project was reviewed by analysing 52 new medical assessments that had taken place during this period. Of these 52 assessments, 30 included documented evidence of exploration of QTc prolongation risk. From these 30 assessments, 21 demonstrated utilisation of the intervention cardiac risk checklist and explored all areas of specific QTc risk factors, while the other nine assessments explored some QT risk factors.

Further analysis of the 30 assessments which had explored QTC 
risk showed that 16 patients prescribed methadone had been identified to be at risk of QTc prolongation. Of these 16 patients with an identified cardiac risk, six were referred to the cardiac assessment clinic in keeping with the guidance in the intervention. In terms of these six referrals for ECGs, only two attended the cardiac assessment clinic and had an ECG performed. For one of these patients, their ECG revealed QTc interval prolongation that allowed the clinician to implement the appropriate management and arrangements for future monitoring.

These results indicate an improvement in the assessment of QTc interval prolongation risk factors, including an increased identification of patients prescribed methadone with a cardiac risk. It is also an indication of an improvement in the management and monitoring of cardiac risk in these patients as BDAS. These results suggest that the intervention has successfully improved awareness within clinician assessments and monitoring of cardiac risk, as well as increasing the support and guidance in these areas of practice.

\section{Lessons and limitations}

This project highlighted the crucial significance of successfully recruiting as many members of the prescribing team as possible to take the amendments to the standard new medical assessment guidance document on board. It particularly indicated the need to engage experienced prescribers with the reasons for an amendment to the assessment process, with an additional new emphasis promoting the assessment and monitoring of cardiac risk in patients prescribed methadone.

It is hoped that prescribers will have heightened awareness of the significance of cardiac risk in methadone prescribing based on the findings of this project. They should also be aware of the potential improvements possible in the rigour of cardiac assessment and monitoring, and how this can be realised by more extensively utilising the interventions resulting from this project. This project revealed how increasing numbers of cardiac risks can be uncovered and appropriately managed, as well as laying the ground for future quality improvement projects to build upon.

The results were limited due to the low incidence of identified cardiac risk cases; the project could be improved by analysing larger samples of patients at the service. This project could have improved its effectiveness in recruiting prescribers to utilise the interventions resulting from this project. A challenging area for cardiac assessment and monitoring in patients that were prescribed methadone was the engagement of these patients in attending their appointments, for example for referrals to the cardiac assessment clinic. Working on improving attendance by this population would be an important area for future quality improvement projects to consider.

\section{Conclusion}

The assessment and management of cardiac risk in patients being prescribed methadone is important for patient safety. QTc interval prolongation is uncommon but potentially life-threatening. There are known risk factors for QTc prolongation which include the prescription of methadone especially at doses above 100 milligrams daily. This project amended the current system at BDAS for the content of medical assessments and monitoring to specifically include a detailed checklist of QTc prolonging risk factors. It encourages and supports those prescribers that are undertaking medical assessments and reviews of patients prescribed methadone to provide a comprehensive assessment and identify any cardiac risk present, as well as providing guidance for managing and monitoring QTc prolongation risk. Implementation of the project highlighted the importance and challenges of recruiting prescribers in utilising amendments to current systems, as well as to the poor attendance for clinics seen in this population of patients prescribed methadone attending an addiction service.

\section{References}

1. Taylor D, Paton C, Kapur S. The Maudsley Prescribing Guidelines in Psychiatry. 2012 11th ed. London: Wiley Blackwell.

\section{Declaration of interests}

Nothing to declare.

\section{Acknowledgements}

Dr Pardeep Grewal, consultant psychiatrist, Barnet Drug and Alcohol Service, Barnet, Enfield, and Haringey Mental Health NHS Trust for the mentorship vital to the success of this quality improvement project. 